\title{
BMJ Open Assessing seropositivity for IgG antibodies against SARS-CoV-2 in Ahmedabad city of India: a cross- sectional study
}

Om Prakash, ${ }^{1}$ Bhavin Solanki, ${ }^{2}$ Jay K Sheth (1) , ${ }^{3}$ Bhavin Joshi, ${ }^{2}$ Mina Kadam, ${ }^{4}$ Sheetal Vyas, ${ }^{3}$ Aparajita Shukla, ${ }^{5}$ Hemant Tiwari, ${ }^{5}$ Sanjay Rathod, ${ }^{4}$ Anil Rajput, ${ }^{4}$ Toral Trivedi, ${ }^{4}$ Vaibhav Ramanuj, ${ }^{5}$ Anand Solanki ${ }^{3}$

To cite: Prakash 0, Solanki B, Sheth JK, et al. Assessing seropositivity for IgG antibodies against SARS-CoV-2 in Ahmedabad city of India: a cross-sectional study. BMJ Open 2021;11:e044101. doi:10.1136/ bmjopen-2020-044101

- Prepublication history for this paper is available online. To view these files, please visit the journal online (http://dx.doi org/10.1136/bmjopen-2020044101).

Received 27 August 2020 Revised 13 December 2020 Accepted 14 December 2020

Check for updates

(c) Author(s) (or their employer(s)) 2021. Re-use permitted under CC BY-NC. No commercial re-use. See rights and permissions. Published by BMJ.

${ }^{1}$ Ahmedabad Municipal Corporation, Ahmedabad, Gujarat, India

${ }^{2}$ Health Department, Ahmedabad Municipal Corporation, Ahmedabad, Gujarat, India ${ }^{3}$ Department of Community Medicine, AMC MET Medical College, Ahmedabad, Gujarat, India

${ }^{4}$ Department of Microbiology, AMC MET Medical College, Ahmedabad, Gujarat, India

${ }^{5}$ Department of Community Medicine, Smt NHL Municipal Medical College, Ahmedabad, Gujarat, India

Correspondence to

Dr Jay K Sheth;

jayksheth@yahoo.com

\section{ABSTRACT}

Objectives To study the percentage seropositivity for SARS-CoV-2 to understand the pandemic status and predict the future situations in Ahmedabad.

Study design Cross-sectional study.

Settings Field area of Ahmedabad Municipal Corporation. Participants More than 30000 individuals irrespective of their age, sex, acute/past COVID-19 infection participated in the serosurvey which covered all the 75 Urban Primary Health Centres (UPHCs) across 48 wards and 7 zones of the city. Study also involved healthcare workers (HCWs) from COVID-19/non-COVID-19 hospitals.

Interventions Seropositivity of IgG antibodies against SARS-CoV-2 was measured as a mark of COVID-19 infection.

Primary and secondary outcomes Seropositivity was used to calculate cumulative incidence. Correlation of seropositivity with available demographic detail was used for valid and precise assessment of the pandemic situation.

Results From 30054 samples, the results were available for 29891 samples and the crude seropositivity is $17.61 \%$. For all the various age groups, the seropositivity calculated between $15 \%$ and $20 \%$. The difference in seropositivity for both the sex group is statistically not significant. The seropositivity is significantly lower (13.64\%) for HCWs as compared with non-HCWs (18.71\%). Seropositivity shows increasing trend with time. Zone with maximum initial cases has high positivity as compared with other zones. UPHCs with recent rise in cases are leading in seropositivity as compared with earlier and widely affected UPHCs.

Conclusions The results of serosurveillance suggest that the population of Ahmedabad is still largely susceptible. People still need to follow preventive measures to protect themselves till an effective vaccine is available to the people at large. The data indicate the possibility of vanishing immunity over time and need further research to cross verify with scientific evidences.

\section{INTRODUCTION}

A new respiratory virus causing SARS-CoV-2 was first reported from China in December 2019 and soon spread throughout the
Strengths and limitations of this study

- Large representative sample of more than 30000 people, including healthcare workers.

- Interesting scientific findings on the seropositivity for IgG antibodies against SARS-COV-2.

- Seropositivity also compared with reported cases.

- Limited demographic detail available to check correlation with seropositivity.

world. ${ }^{12}$ The WHO declared it as pandemic and named the disease caused by this virus as COVID-19. ${ }^{3}$ Being a newly identified virus, the scientific community is largely unaware of the natural history and the immune response developed after the COVID-19 infection. ${ }^{4}$ Since the virus is novel in origin, the initial seropositivity in the population is nil. So, a population-based serosurvey can help in estimating cumulative incidence of a novel infection as well as extent of the infection in the community. ${ }^{5}$ WHO also recommends monitoring of seroprevalence over time for anticipating disease dynamics and planning an adequate public health response. ${ }^{6}$ The sample size in such sample survey should be large enough to get reliable parameters sufficient enough to draw conclusions and future public health actions. ${ }^{7}$

Serological tests and the seroepidemiology greatly help in understanding the disease transmission, population susceptibility as well as the public health measures to be followed. ${ }^{8}$ Since the test for COVID-19 infection turns out to be positive even in an asymptomatic patient, restricting the serological testing only to symptomatic individual will not give a real picture. ${ }^{9}$ On the other hand, a field level population-based testing will give a better assessment of disease situation and the specific immunity following its infection. 
While the positive results indicate what proportion of the testing population has developed immunity, those with negative result gives hint about the proportion of susceptible population.

India being the second most populous country with high population density is at high risk from COVID-19 pandemic. Ahmedabad city was among the first few cities severely affected by the spread of the pandemic. Ahmedabad city of India, having 7 million population, was one of the earliest cities to witness the high case load in the initial months of the pandemic in India. Ahmedabad had approximately 16360 reported COVID-19 cases and 1184 reported COVID-19 deaths before start of this study. A serosurveillance study by the Indian Council of Medical Research (ICMR) in the containment zones of the city carried out on 28-29 May 2020 had reported 55\% seroprevalence, the highest among all the containment zones from all the major cities of India. ${ }^{10}$ Central zone of the city was one of the highly affected area which was kept under area containment for more than a month from 26 April 2020. With such high number of cases and transmission, Ahmedabad was ideally suited to study the percentage seropositivity in general population to help understand the pandemic status and predict the future situations.

\section{Aim}

- To analyse and study the available data related to COVID-19 seropositivity in Ahmedabad City

\section{Objectives}

- To calculate the seroprevalence of IgG antibodies to COVID-19 in the general population in Ahmedabad.

- To correlate the seropositivity with various factors for better understanding of the pandemic situation.

- To identify the immunity status for valid and precise predictions for the future.

\section{METHODOLOGY}

Looking to monitor the pandemic, understand its present situation and to take appropriate corrective public health measures, the ICMR issued directives to all the state governments to carry out IgG Elisa test for serosurveys along with ILI \& SARI Surveillance. ${ }^{11}$ The primary purpose of this was to understand the proportion of population exposed to SARS-CoV-2 including asymptomatic individuals. The health department of the local municipality of Ahmedabad-Ahmedabad Municipal Corporation (AMC), from the state of Gujarat, India, carried out a large-scale population-based serological survey for IgG antibodies against SARS-CoV-2. This was one of the earliest primary situational analyses of the immune status against the SARS CoV-2 infection from India.

Ahmedabad city is divided into 48 wards distributed across 7 zones. There are 75 Urban Primary Health Centres (UPHCs) which cater the primary healthcare services to the local population. To get the real status of immunity, authorities of AMC preferred a field level serological study over case load or case density-related sampling. At the UPHC, a trained laboratory technician is available along with necessary basic laboratory support for handling the sample, and so, the sample collection in the field area was managed by the staff posted at the UPHCs. For enrolment in the study, convenience sampling was followed at the level of UPHCs. The field level healthcare workers (HCWs) facilitated the enrolment of the willing individuals for the purpose of the study. An effort was made to cover a wide variety of people of different age groups from both the gender and falling into various categories without any exception. Thus, the inclusion criteria allowed inclusion of all irrespective of age, sex, acute/past COVID-19 infection. Exclusion criteria included refusal to give informed verbal consent or any contraindication to venipuncture.

AMC manages one dedicated COVID-19 hospital which is attached with a medical college. There are two other non-COVID-19 municipal general hospitals which are attached with medical colleges. As many of the health workers working at these hospitals have developed COVID-19 infection during the first 3 months of the pandemic, the authorities were concerned about their immune status and invited willing HCWs to enrol from the three hospitals under their administrative control to participate. So, apart from the 75 UPHCs, enrolment of the HCWs was also carried out at these three hospitals for the purpose of antibody testing.

An informed verbal consent was taken from all the participants before enrolment. Strict confidentiality was ensured at all the levels. Looking to the sample handling capacity at the UPHC and the testing capacity of the laboratories, the study continued over a period of approximately 3 weeks (from 16 June to 7 July). For the purpose of quality testing and reliability of results, only medical college laboratories were considered. Accredited private laboratories with all necessary equipment and facilities, which routinely undergo external quality assurance, were also considered for timely results of the large number of samples.

'Covid Kavach' (anti-SARS CoV-2 IgG antibody detection capture ELISA) kits developed and manufactured by Zydus Diagnostics, validated by National Institute of Virology, Pune, India and approved for use by the ICMR was used for the purpose of this study after due approval. As per the validation reports, the kits have a sensitivity of $92.37 \%$ and a specificity of $97.9 \%{ }^{12}$ So, with very high level of sensitivity and specificity it may be noted that the results received through this testing kit are highly reliable and the kit is permitted for use in serosurveillance of SARS-CoV-2. The manufacturer reported no crossreactivity with other viruses in the serum from real-time RTqPCR confirmed patients of various other infections. Testing procedures were followed as per the manufacturer's instructions. For each plate, samples with optical density $(\mathrm{OD})$ value more than the cut-off value and positive/negative $(\mathrm{P} / \mathrm{N})$ ratio more than 1.5 were considered as positive. Samples with OD value of 10 per cent \pm ranges of the cut-off were considered to be indeterminate. The 


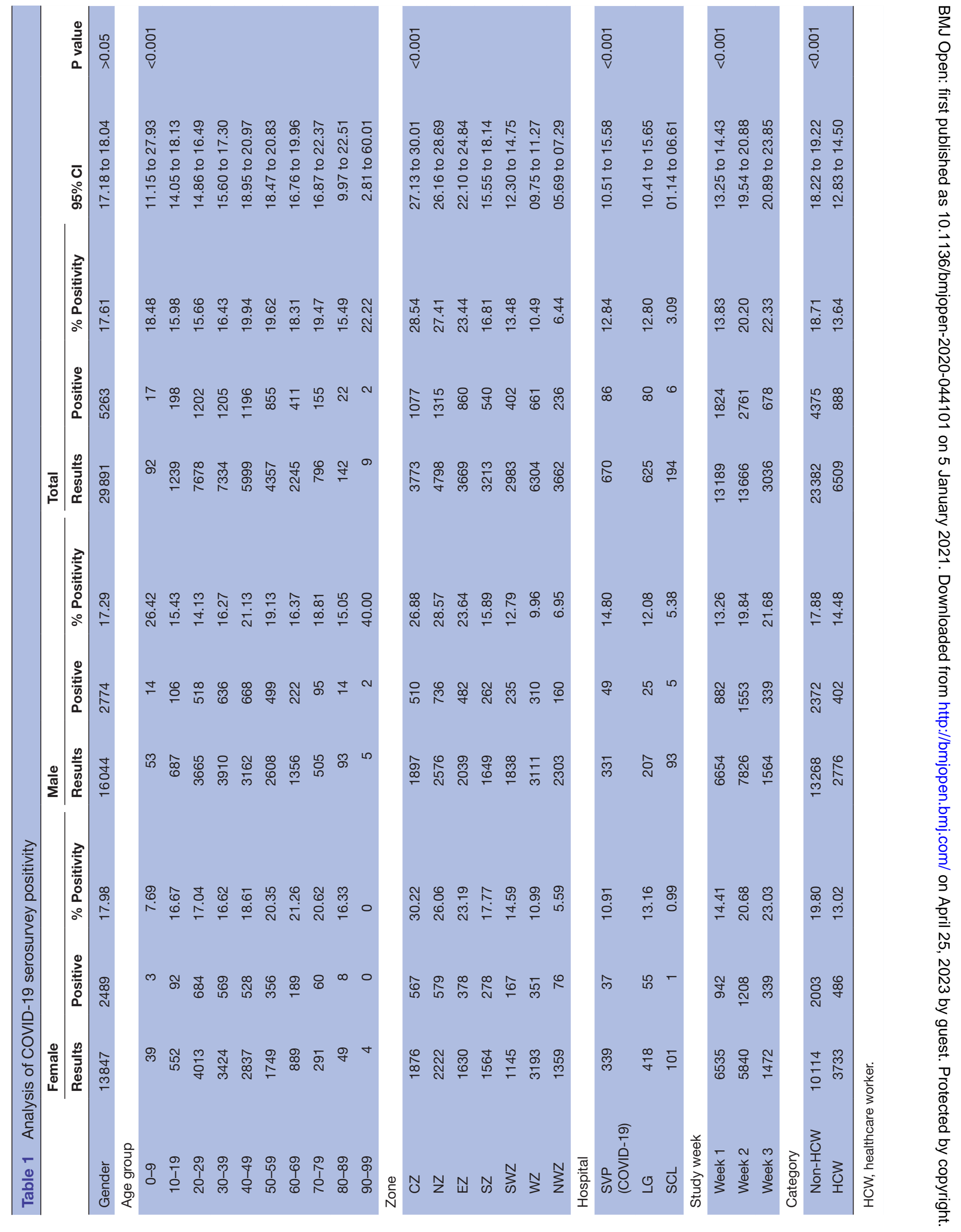


$\mathrm{P} / \mathrm{N}$ ratio was defined as the ratio of average $\mathrm{OD}$ value of the positive control divided by the average OD of the negative control. The cut-off OD value was calculated as the average $\mathrm{OD}$ value of negative control +0.2 .

AMC had collected the data of the enrolled participants in a simple brief format with minimum basic primary demographic information. An in-depth analysis of available data was crucial for valid and precise estimation of immunity status and for guiding the authorities for taking appropriate public health measures in a timely manner. So, the faculty members involved in corona control room activities, field monitoring and supervision as well as antibody testing were invited to critically analyse the available data and draw scientific conclusion on the level of seropositivity. Approval to study and analyse the available data were granted by the Institutional Review Board. We tried to find out the crude positivity as an indicator for the level of cumulative incidence and compared it with various other factors. We shared the results with the concerned authorities to take appropriate public health measures for larger benefit of the population. We herewith share the results for the detailed insight by the scientific community.

Patient and public involvement: patients were not directly involved in the planning or implementation of the serosurveillance. However, these are carried out by the administrators of the local self-governance (municipal corporation) and were carried out for their benefit and determining their immunity status. The results were declared to the local media through press conference and were widely publicised on social media through executive summary and published by local daily newspapers as well.

\section{RESULTS}

A total of 30054 blood samples were collected for the purpose of this study, of which 1511 samples were collected from the 3 hospitals while the remaining 28543 samples were collected from the 75 UPHCs. A total of 163 samples were rejected for various reasons. Results were available for the remaining 29891 samples (table 1). A total of 24197 tests were negative while 431 test results were reported as indeterminate. Thus, a total of 5263 results were positive for the specific antibodies against COVID-19 giving an overall crude positivity of about $17.61 \%$ (figure 1 ).

There were 16135 men and 13919 women enrolled in the study and results were available for 29891 individuals. A total of 2774 from 16044 men were tested positive giving the positivity rate of $17.29 \%$. A total of 2489 out of 13847 women were tested positive giving the positivity rate of $17.98 \%$. Thus, the percentage positivity is slightly higher among women as compared with the men but the difference is statistically not significant $(p>0.05)$.

The age-wise analysis shows that the age of the sample population ranges from <1 year to 100 years with an average of 39.10 years with an SD of 14.54 years. Among the sample, the mean age of women is 37.70 years with an

\section{Result of Sero-surveillance}

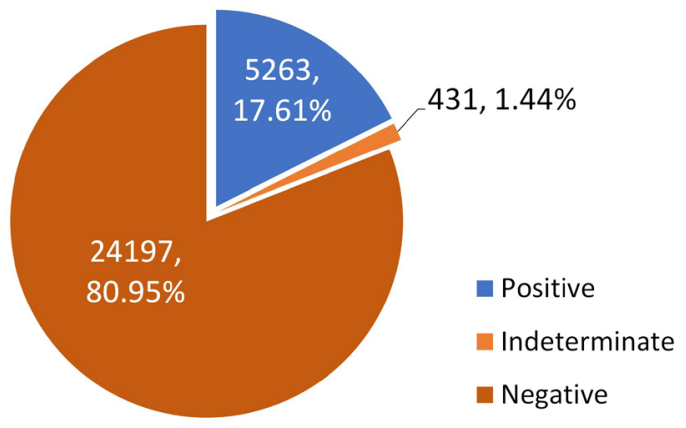

Figure 1 Result of serosurveillance.

SD of 13.93 years, whereas the mean age of men is 40.25 years with an SD of 14.88 years. Considering the seropositive, the mean age for women is 38.69 years with an SD of 14.16 years whereas that of men is 41.41 years with an SD of 14.51 years.

The age groupwise analysis of total tests and positive tests when compared to calculate per cent positivity (figure 2) shows that the positivity in various age groups is between $15 \%$ and $20 \%$. Considering the gender variation also for different age groups (figure 3), the per cent positivity for both the genders is seen between $15 \%$ and $20 \%$ for most of the age groups. The age groupwise distribution is statistically significant.

UPHC-wise positivity was compared for the first 10 UPHCs with highest positivity (table 2). The comparison of zone-wise positivity with cases (table 3 ) shows that the zone most affected by COVID-19, that is, central zone with maximum initial cases had the highest positivity of $28.54 \%$. Thus, the positive antibody status correlates with the documented evidence of high case load particularly in the first 2 months of the pandemic in Ahmedabad.

Our study included HCWs from field area as well as COVID-19/non-COVID-19 hospitals. With 888 seropositives out of 6509 HCWs, the seropositivity among HCWs is $13.64 \%$. On the other hand, seropositivity among nonHCWs was $18.71 \%$. Seropositivity among non-HCW is significantly higher as compared with the HCWs.

We also tried to compare positivity with days of our study period, which showed wide variation. To remove the effect of daily variation we tried to study the linear trend (figure 4). We tried to calculate the weekly seropositivity for the 3-week study duration (table 1), which shows a seropositivity of $13.83 \%, 20.20 \%$ and $22.33 \%$, respectively. Zone-wise seropositivity and its comparison with cases per 10000 population (figure 5) and case density (figure 6) show that the positivity correlates with the cases in most zones but are strikingly low in central zone.

\section{DISCUSSION}

The present study is one of the biggest studies conducted in the world in terms of sample population ratio to find 


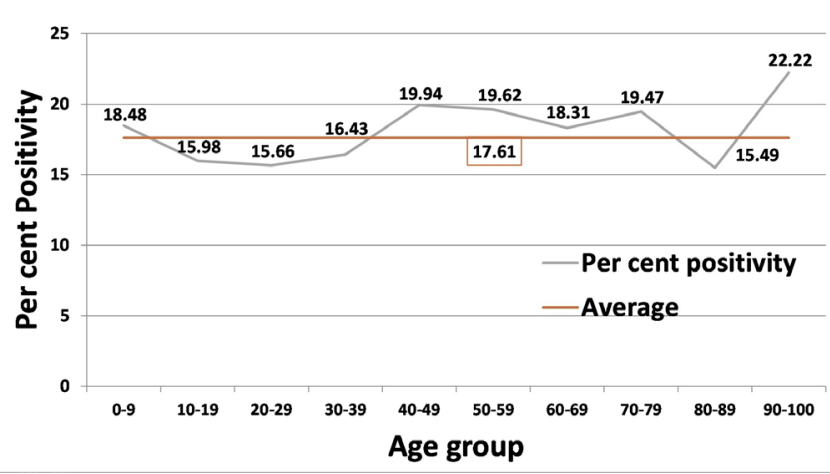

Figure 2 Age groupwise seropositivity.

out seropositivity in general population. This study has 4770 samples per million population in comparison to Spanish study where the sample population ratio was 1302 samples per million population and the US study where the sample population ratio was 255 samples per million population. ${ }^{13}{ }^{14}$ With $17.61 \%$ seropositivity our results are consistent with other studies showing that even in the areas highly affected by SARS CoV-2 during this pandemic have shown very low level of seropositivity. ${ }^{8}$ Antibodies do take some time to develop after an infection, approximately 1-3 weeks, with an average of 2 weeks (14 days). ${ }^{1516}$ So, we can say that the rate of antibody positivity reflects the case scenario about 14 days prior to the study. A national seroprevalence study from India, completed a few days before the present study, documented the national seroprevalence of just $0.73 \%$ (95\% CI $0.34 \%$ to $1.13 \%$ ). The vast difference reflected in our study justifies our statement for the situation in Ahmedabad during the initial 3 months of the pandemic. ${ }^{17} \mathrm{On}$ the other hand, the study by ICMR found $55 \%$ seroprevalence in the containment zone. ${ }^{10}$

The difference in the positivity for both the sex group is statistically not significant and this finding is similar to other studies from Spain and USA where the studies have found no significant difference between the genders for COVID-19 antibodies. ${ }^{7818}$ Comparatively less samples with wide variation in positivity at the extremes of age group, that is, 0-9 years and 90-99 years for both the gender groups may be the reason for having a statistically

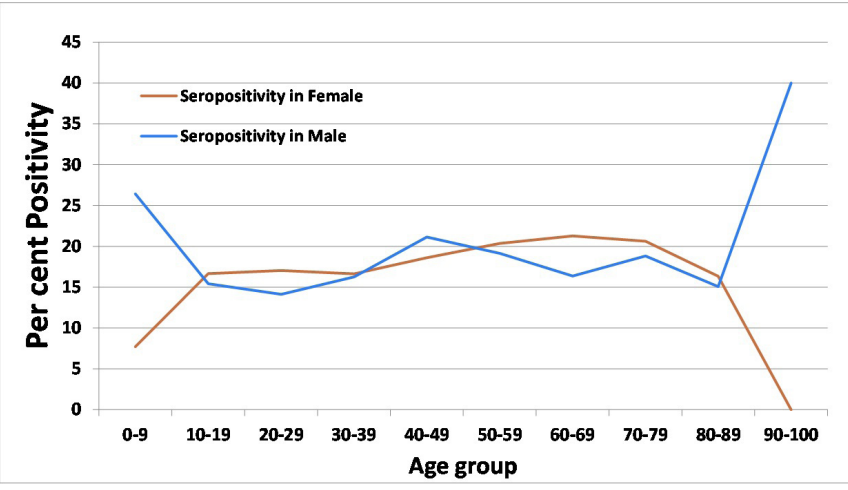

Figure 3 Age group and sex-wise seropositivity.
Table 2 Top 10 Urban Primary Health Centre (UPHC) with highest seropositivity results

\begin{tabular}{lllcl}
\hline UPHC of AMC & Total & Results & Positive & Positivity \\
\hline Meghaninagar (NZ) & 119 & 119 & 52 & 43.70 \\
\hline Gomtipur (EZ) & 308 & 300 & 118 & 39.33 \\
\hline Rajpur (EZ) & 383 & 383 & 138 & 36.03 \\
\hline Naroda road (NZ) & 170 & 170 & 61 & 35.88 \\
\hline Indiacolony (NZ) & 806 & 802 & 280 & 34.91 \\
\hline Bapunagar (NZ) & 316 & 316 & 110 & 34.81 \\
\hline Kubernagar (NZ) & 480 & 477 & 166 & 34.80 \\
\hline Saraspur-rakhial (NZ) & 401 & 401 & 127 & 31.67 \\
\hline Jamalpur (CZ) & 429 & 428 & 135 & 31.54 \\
\hline Shahpur (CZ) & 394 & 394 & 124 & 31.47 \\
\hline
\end{tabular}

AMC, Ahmedabad Municipal Corporation.

significance difference for the age group-wise seropositivity. Majority of the HCWs in the study were from the field area-working at the UPHCs. A statistically significant low seropositivity among HCW as compared with non-HCW indicates that they are better protected as compared with general population, particularly when the epidemic has progressed to infect a large number of people from general population.

Our results also show that the UPHCs with high number of cases have higher positivity. However, UPHCs with high number of cases in the early phase of the pandemic have low seropositivity as compared with the UPHCs with higher number of cases during the later part of the pandemic (with more cases in the recent past). In Ahmedabad, the initial cases were more from the central zone followed by south zone. Then, gradually due to extensive containment measures the cases from central zone reduced and cases started appearing more from the east and north zones. It can be seen that the highest seropositivity was found in the individuals from east zone and north zone. This is on a slightly higher side than that from the UPHCs of the central zone, which had the overall highest number of cases. Strikingly the worst and first affected UPHCs of the central zone have lesser positivity. The first eight UPHCs with the highest positivity are from north zone and east zone (having higher cases in the later part of the pandemic period so far) than that of central zone (having maximum population density and highest cases in the very first phase of pandemic period). This might be pointing towards the fact that the immunity developed after a successful recovery from COVID-19 infection may not be lasting enough. Although the same is also reported by other studies, we need more studies and longer follow-up to cross verify this aspect and to bring out the scientific fact related with the post-COVID-19 immunity. ${ }^{19-21}$

Day to day variation in positivity may be dependent on the proportion of different population groups (eg, cases, contacts, super spreaders, symptomatic, asymptomatic, HCWs, etc) covered on that day. However, the trend 
Table 3 Zone-wise comparison of seropositivity and COVID-19 cases

\begin{tabular}{|c|c|c|c|c|c|c|c|c|}
\hline Zone & Population & $\begin{array}{l}\text { Area } \\
\left(\mathrm{km}^{2}\right)\end{array}$ & $\begin{array}{l}\text { Cases } \\
\text { (as on } 15 \text { June } \\
2020 \text { ) }\end{array}$ & $\begin{array}{l}\text { Case density } \\
\left(\text { cases } / \mathbf{k m}^{2}\right)\end{array}$ & $\begin{array}{l}\text { Cases } \\
\text { per } 10000 \\
\text { population }\end{array}$ & Results & positive & $\begin{array}{l}\text { Per cent } \\
\text { positivity }\end{array}$ \\
\hline $\mathrm{CZ}$ & 683089 & 18.33 & 3923 & 214.0 & 57.43 & 3773 & 1077 & 28.54 \\
\hline SZ & 1081996 & 88.65 & 3276 & 37.0 & 30.28 & 3213 & 540 & 16.81 \\
\hline NZ & 1090409 & 64.92 & 2889 & 44.5 & 26.49 & 4798 & 1315 & 27.41 \\
\hline EZ & 1425254 & 73.2 & 2532 & 34.6 & 17.77 & 3669 & 860 & 23.44 \\
\hline SWZ & 524970 & 61.86 & 871 & 14.1 & 16.59 & 2983 & 402 & 13.48 \\
\hline NWZ & 807539 & 91.36 & 772 & 8.5 & 9.56 & 3662 & 236 & 6.44 \\
\hline
\end{tabular}

suggests that the positivity slowly increases with time. The same is the conclusion from the week-wise positivity calculated for the 3-week study duration. Both of these findings show that the positivity increases with time and thus correlates with increasing number of cumulative cases.

When we compare cases per million population with seropositivity (table 3, figure 5) it was found that most affected central zone with highest cases ( 5743 per million population) also had highest seropositivity of $28.54 \%$. However, the areas of recent infection, like north zone and east zone, have higher seropositivity (27.41\%-23.44\%, respectively) despite having lower cases (2649 and 1777 cases per million population, respectively) than the south zone. The comparative low positivity as compared with high case per million population may be largely due to the sampling method followed, which was independent of the cases from that zone. We also tried to compare the case density (cases per $\mathrm{km}^{2}$ ) with seropositivity (table 3 , figure 6 ). Here, also the findings suggest that the first and worst affected central zone with highest case density had highest seropositivity. Areas of recent infection like north zone and east zone have higher seropositivity of $27.41 \%$ and $23.44 \%$, respectively (much higher than that of south zone, ie, $16.81 \%$ ) despite having case density comparative with that of south zone (44.5 and 34.6 cases per $\mathrm{km}^{2}$, respectively, as against 37.0 cases per $\mathrm{km}^{2}$ in south zone). Both these comparisons indicate the possibility

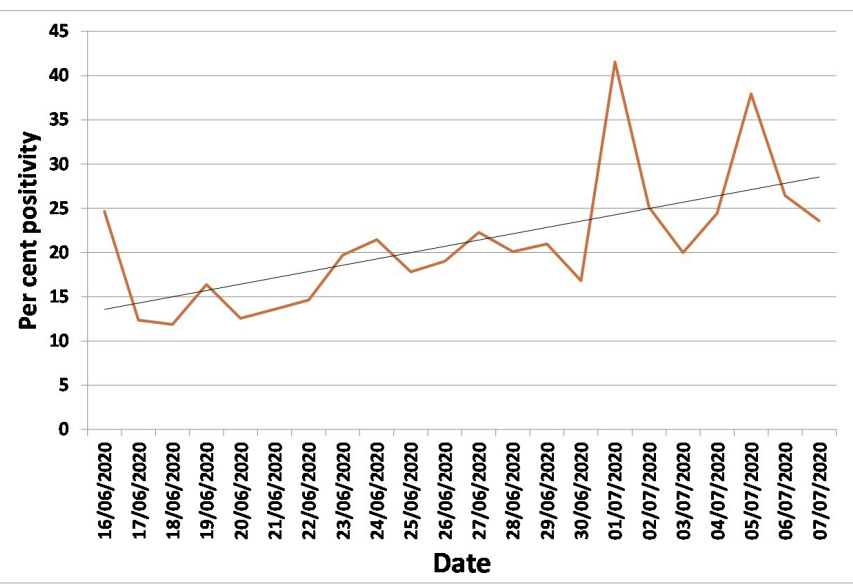

Figure 4 Daywise seropositivity. of vanishing positivity with the passing of time. However, we need more in-depth scientific research to find out the reason behind this paradox.

Looking at the positivity rate at the COVID-19/nonCOVID-19 hospitals, it is observed that the highest positivity (12.84\%) is seen at the SVP hospital which is a dedicated COVID-19 hospital right from the start of the corona pandemic in the city. The overall positivity of $12.84 \%$ is still lower than that of the crude positivity of the general population, that is, $17.61 \%$. This may be due to the fact that all the HCWs in a dedicated COVID-19 hospital are very well protected with Personal Protective Equipment (PPE) and other safety measures.

LG hospital is a non-COVID-19 hospital attached with another medical college run by the AMC. The positivity rate at LG is also quite similar to SVP hospital with $12.80 \%$ positivity. Although LG hospital is not a dedicated COVID-19 hospital, still the positivity rate here is quite similar to that of the COVID-19 hospital. Inspite of being a non-COVID-19 hospital, there were multiple occasions in the previous 2 months when large number of HCWs turned out to be positive and many HCWs were isolated/quarantined. A lot of patients from the containment zone reported here for non-COVID-19 complains but were tested and reported positive. The authorities were even forced to close down the hospital once for about 7 days for improving the sanitisation measures and improving the implementation of various Standard Operating Protocols (SOPs). As compared with

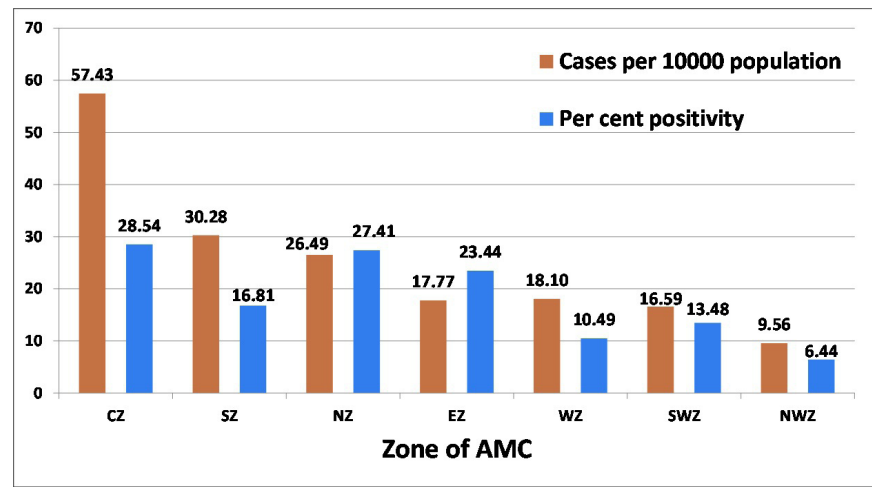

Figure 5 Comparison of seroprevalence with zone-wise cases. AMC, Ahmedabad Municipal Corporation. 


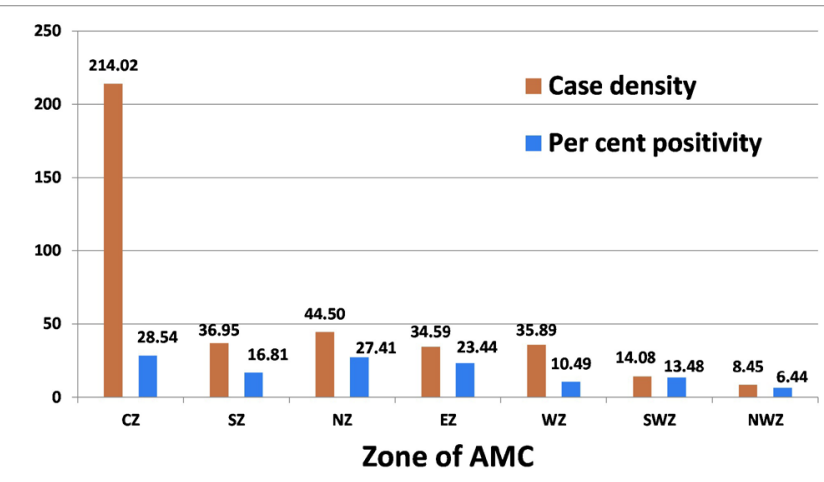

Figure 6 Comparison of seroprevalence with case density. AMC, Ahmedabad Municipal Corporation.

the above two hospitals, the other non-COVID-19 hospital (SCL hospital) did not have many COVID-19 cases and the low positivity $(3.09 \%)$ at this hospital also correlates with the data.

\section{Limitation of our study}

The study followed convenience sampling at the level of UPHC. Some of the collected data (eg, symptomatology of the enrolled individual) was not completely available for data analysis.

\section{CONCLUSION}

This study to assess the seropositivity during the COVID-19 pandemic from Ahmedabad, India is one of the first few population-based studies from India with a large sample size and a very high sample-population ratio. As of June 2020, the level of COVID-19 seropositivity in Ahmedabad city, India is $17.61 \%$. In view of these findings with the absence of an evidence of lifelong immunity after COVID-19 infection, it can be concluded that the population of Ahmedabad is still largely susceptible. As of now, we cannot rely on this level of immunity to protect and the preventive measures need to be strongly relied on till an effective vaccine is provided to the people at large. There is no gender difference in seropositivity but the seropositivity is significantly associated with the risk of COVID-19 infection in the area. Although the seropositivity slowly increases with time, there are also indications that these IgG may not be long lasting. Further in-depth scientific studies are required to give more insight for the future predictions.

Acknowledgements The authors are extremely thankful to respected Dr Rajiv Kumar Gupta, IAS (Additional Chief Secretary, Government of Gujarat) and Mr Mukesh Kumar, IAS (Municipal Commissioner, Ahmedabad) for their whole hearted support. This study would not have been possible without the financial support from the authorities of Ahmedabad Municipal Corporation. They acknowledge the full support from the field level healthcare workers (Corona warriors) who put in great efforts to perform their duties as well as sample collection after informed verbal consent particularly in a COVID-19 pandemic situation. All the Zonal Deputy Health Officers, Deputy Health Officer (Epidemic), Assistant Health Officers and Medical officers of the Urban Primary Health Centres extended full support in conducting the serosurveillance. They are thankful to all the medical and paramedical support staff posted at the laboratories for their contribution in timely testing such large number of samples with accuracy and quality. Finally, they are indebted to all the patients including healthcare workers whose willingness and support has generated the much-desired data for the study.

Contributors BS under the guidance of OP planned and carried out the serosurveillance. Testing of the samples and reporting were managed by SR, AR and TT under the leadership of MK. Data analysis was carried out by JKS, SV, ASh, BJ, VR and ASo while the statistical analysis was done by HT. Primary manuscript was prepared by OP and JKS and equally contributed by all the other coauthors. All authors contributed to the interpretation of data and approved the final manuscript after critical review.

Funding Health Department of Ahmedabad Municipal Corporation carried out the study as part of COVID-19 pandemic response.

Competing interests None declared.

Patient and public involvement Patients and/or the public were not involved in the design, or conduct, or reporting, or dissemination plans of this research.

Patient consent for publication Not required.

Ethics approval The authors have received permission for the study from the Institutional Review Board (IRB) of the AMC MET Medical College, Ahmedabad.

Provenance and peer review Not commissioned; externally peer reviewed

Data availability statement Upon reasonable request for data sharing to the corresponding author, our data are accessible to researchers.

Open access This is an open access article distributed in accordance with the Creative Commons Attribution Non Commercial (CC BY-NC 4.0) license, which permits others to distribute, remix, adapt, build upon this work non-commercially, and license their derivative works on different terms, provided the original work is properly cited, appropriate credit is given, any changes made indicated, and the use is non-commercial. See: http://creativecommons.org/licenses/by-nc/4.0/.

ORCID iD

Jay K Sheth http://orcid.org/0000-0002-9598-4473

\section{REFERENCES}

1 Munster VJ, Koopmans M, van Doremalen N, et al. A novel coronavirus emerging in China - key questions for impact assessment. N Engl J Med Overseas Ed 2020;382:692-4.

2 Li Q, Guan X, Wu P, et al. Early transmission dynamics in Wuhan, China, of novel coronavirus-infected pneumonia. N Engl J Med 2020;382:1199-207.

3 Cucinotta D, Vanelli M. WHO Declares COVID-19 a pandemic. Acta Biomed 2020;91:157-60.

4 Lipsitch M, Swerdlow DL, Finelli L. Defining the epidemiology of Covid-19 - studies needed. N Engl J Med Overseas Ed 2020;382:1194-6.

5 GeurtsvanKessel CH, Okba NMA, Igloi Z, et al. An evaluation of COVID-19 serological assays informs future diagnostics and exposure assessment. Nat Commun 2020;11:3436.

6 World Health Organization. Population-Based age-stratified seroepidemiological investigation protocol for COVID-19 virus infection, 2020. Available: https://apps.who.int/iris/handle/10665/ 331656 [Accessed 9 Nov 2020].

7 Hanage W, Qiu X, Kennedy-Shaffer L. Snowball sampling study design for serosurveys in the early COVID-19 pandemic.

8 Bryant JE, Azman AS, Ferrari MJ, et al. Serology for SARS-CoV-2: Apprehensions, opportunities, and the path forward. Sci Immunol 2020;5. doi:10.1126/sciimmunol.abc6347. [Epub ahead of print: 19 May 2020].

9 Velavan TP, Meyer CG. The COVID-19 epidemic. Trop Med Int Health 2020;25:278-80.

10 The Economic Times. Prevalence of Covid-19 infection, at 55\%, is the highest in Ahmedabad containment zones: Sources. Available: https://economictimes.indiatimes.com/industry/healthcare/biotech/ healthcare/prevalence-at-55-highest-in-abad-containment-zones/ articleshow/77073909.cms?from $=\mathrm{mdr}$

11 ICMR. ICMR advises States to conduct sero-survey to measure Coronavirus exposure in the population using IgG ELISA Test. Available: https://main.icmr.nic.in/sites/default/files/press_realease_ files/ICMR_PR_IgG_Elisa_30052020.pdf

12 Sapkal G, Shete-Aich A, Jain R, et al. Development of Indigenous IgG ELISA for the detection of anti-SARS-CoV-2 IgG. Indian J Med Res 2020;151:444.

13 Pollan M, Perez-Gomez B, Pastor-Barriuso R. A population-based seroepidemiological study of SARS-CoV-2 in Spain (ENE-COVID). 
14 Medrxiv. Cdc: large scale geographic sero prevalence surveys. Available: https://www.medrxiv.org/content/10.1101/2020.06.25. 20140384v1.full.pdf [Accessed 10 Aug 2020].

15 Tripathi SC, Deshmukh V, Patil A, et al. COVID 19 diagnostic multiplicity and its role in community surveillance and control. Infez Med 2020;28:18-28.

16 Zhao J, Yuan Q, Wang $\mathrm{H}$, et al. Antibody responses to SARS-CoV-2 in patients with novel coronavirus disease 2019. Clin Infect Dis 2020;71:2027-34.

17 Murhekar MV, Bhatnagar T, Selvaraju S, et al. Prevalence of SARSCoV-2 infection in India: findings from the National serosurvey, MayJune 2020. Indian J Med Res 2020;152:48.
18 Stringhini S, Wisniak A, Piumatti G, et al. Seroprevalence of antiSARS-CoV-2 IgG antibodies in Geneva, Switzerland (SEROCoVPOP): a population-based study. Lancet 2020;396:313-9.

19 Seow J, Graham C, Merrick B, et al. Longitudinal observation and decline of neutralizing antibody responses in the three months following SARS-CoV-2 infection in humans. Nat Microbiol 2020;5:1598-607.

20 Sethuraman N, Jeremiah SS, Ryo A. Interpreting diagnostic tests for SARS-CoV-2. JAMA 2020;323:2249-51.

21 Rosado J, Pelleau S, Cockram C. Serological signatures of SARSCoV-2 infection: implications for antibody-based diagnostics, 2020. 